\title{
Symmetries and Conservation Laws for Some Compacton Equation
}

\author{
M. L. Gandarias, M. S. Bruzón, and M. Rosa \\ Departamento de Matemáticas, Universidad de Cádiz, Puerto Real, 11510 Cádiz, Spain \\ Correspondence should be addressed to M. S. Bruzón; m.bruzon@uca.es
}

Received 13 May 2015; Revised 9 July 2015; Accepted 2 August 2015

Academic Editor: Martino Bardi

Copyright (c) 2015 M. L. Gandarias et al. This is an open access article distributed under the Creative Commons Attribution License, which permits unrestricted use, distribution, and reproduction in any medium, provided the original work is properly cited.

\begin{abstract}
We consider some equations with compacton solutions and nonlinear dispersion from the point of view of Lie classical reductions. The reduced ordinary differential equations are suitable for qualitative analysis and their dynamical behaviour is described. We derive by using the multipliers method some nontrivial conservation laws for these equations.
\end{abstract}

\section{Introduction}

Solitons and compactons are two kinds of nonlinear waves. They play a vital role in all ramifications of science and technology and are used as constructive elements to formulate the complex dynamical behaviour of wave systems throughout science: from hydrodynamics to nonlinear optics, from plasmas to shock waves, from tornados to the Great Red Spot of Jupiter, and from tsunamis to turbulence. More recently, solitons and compactons are of key importance in the quantum fields and nanotechnology specially in nanohydrodynamics.

It is known that while the impact of typical nonlinearity does not change much with spatial dimension, the spreading effectiveness of dispersive mechanisms increases. Thus a well-balanced one-dimensional model becomes less balanced in higher dimensions. This in general greatly reduces the prevalence of robust solitonic structures in higher spatial dimensions. The compactons, solitons with a compact support, were introduced by Rosenau and Hyman [1] over twenty years ago via the $K(m, n)$ model equation

$$
u_{t}+\left(u^{m}\right)_{x}+\left(u^{n}\right)_{x x x}=0, \quad m, n>1,
$$

and symmetry reductions and exact solutions were derived in $[2,3]$. The conservation laws for the compacton $K(2,2)$ equation and the compacton $K(3,3)$ equation were constructed in [4] by utilizing the multiplier approach. In [4], only multipliers of the form $A(t, x, u)$ were considered since, according to the authors, the higher order multipliers determining equations are too complicated and cannot be separated manually.

In [5] Rosenau and Oron have explored the impact of several prototypical types of a nonconvex convection on a formation of compact patterns. This is done via the simple model with cubic dispersion and some variations on a nonlinear modified dispersion

$$
\begin{aligned}
u_{t}+\left(u^{3}-u^{2}\right)_{x}+\left[u\left(u^{2}\right)_{x x}\right]_{x} & =0, \\
u_{t}+\left(u^{3}-u^{2}\right)_{x}+\left(u^{2}\right)_{x x x} & =0 .
\end{aligned}
$$

They found that unlike the $K(n, n)$ compactons that have a universal, speed-independent width, the present compactons width depends on their velocity. In a recent paper [6], we proved that some equations with compacton solutions and cubic dispersion are both nonlinearly self-adjoint, and we have determined some nontrivial conservation laws.

It is known that conservation laws play a significant role in the solution process of an equation or a system of differential equations. Although not all of the conservation laws of partial differential equations (PDEs) may have physical interpretation they are essential in studying the integrability of the PDEs.

For variational problems, the Noether theorem [1] can be used for the derivation of conservation laws. For nonvariational problems there are different methods for the construction of conservation laws. In $[7,8]$, Anco and Bluman 
gave a general algorithmic method to find all conservations laws for evolution equations like (2) and (3). In [9], a special method has been introduced by Ibragimov. This method, which does not require the existence of Lagrangians, is based on the concept of adjoint equations for nonlinear equations and avoids the integrals of functions. The concept of strictly self-adjoint equations has been generalized [10-12]. After Ibragimov's results, several papers appeared to be concerned with self-adjointness and its applications to PDEs [13-24]. This method is a special case of the formula presented in [7].

In this work we will apply Lie classical method to (2) and (3) in order to obtain exact solution, as well as to derive conservation laws for these equations by using the multipliers method.

\section{Symmetry Reductions and Exact Solutions}

In this section, we perform Lie symmetry analysis for (2) and (3). Let us consider a one-parameter Lie group of infinitesimal transformations in $(x, t, u)$ given by

$$
\begin{aligned}
& x^{*}=x+\varepsilon \xi(x, t, u)+\mathcal{O}\left(\varepsilon^{2}\right), \\
& t^{*}=t+\varepsilon \tau(x, t, u)+\mathcal{O}\left(\varepsilon^{2}\right), \\
& u^{*}=u+\varepsilon \phi(x, t, u)+\mathcal{O}\left(\varepsilon^{2}\right),
\end{aligned}
$$

where $\varepsilon$ is the group parameter. Then one requires that this transformation leaves the set of solutions of (2) and (3) invariant. This yields to the overdetermined, linear system of eleven equations for the infinitesimals $\xi(x, t, u), \tau(x, t, u)$, and $\phi(x, t, u)$. The associated Lie algebra of infinitesimal symmetries is the set of vector fields of the form

$$
\mathbf{v}=\xi \frac{\partial}{\partial x}+\tau \frac{\partial}{\partial t}+\phi \frac{\partial}{\partial u}
$$

Having determined the infinitesimals, the symmetry variables are found by solving the invariant surface condition

$$
\Phi \equiv \xi \frac{\partial u}{\partial x}+\tau \frac{\partial u}{\partial t}-\phi=0
$$

From the determining system for (2), we get that $\xi=\xi(x, t)$, $\tau=\tau(t)$, and $\phi=\phi(x, t, u)$, where $\xi$, $\tau$, and $\phi$ must satisfy the following equations:

$$
\begin{aligned}
& -3 u \xi_{x}+\tau_{t} u+2 \phi=0, \\
& -3 u \xi_{x x}+3 \phi_{u x} u+4 \phi_{x}=0, \\
& -2 u^{2} \xi_{x x x}-3 u^{2} \xi_{x}+2 u \xi_{x}-\xi_{t}+3 \tau_{t} u^{2}+6 \phi_{u x x} u^{2} \\
& \quad-2 \tau_{t} u+8 \phi_{x x} u+6 \phi u-2 \phi=0, \\
& -12 u \xi_{x}+3 \phi_{u u} u^{2}+4 \tau_{t} u+4 \phi_{u} u+4 \phi=0, \\
& -4 u \xi_{x x}+3 \phi_{u u x} u^{2}+8 \phi_{u x} u+3 \phi_{x}=0, \\
& -3 \xi_{x}+\phi_{u u u} u^{2}+4 \phi_{u u} u+\tau_{t}+2 \phi_{u}=0 .
\end{aligned}
$$

After solving the determining equations for $\xi, \tau$, and $\phi$, we obtain the two-dimensional Lie algebra spanned by the following Lie point symmetry generators admitted by (2):

$$
\begin{aligned}
& \mathbf{v}_{1}=\frac{\partial}{\partial x} \\
& \mathbf{v}_{2}=\frac{\partial}{\partial t}
\end{aligned}
$$

In this section, we obtained the reduction of (2) to ODEs using the generator $\lambda \mathbf{v}_{\mathbf{1}}+\mu \mathbf{v}_{\mathbf{2}}$. We have the similarity variable and similarity solution

$$
\begin{aligned}
& z=\mu x+\lambda t \\
& u=h(z)
\end{aligned}
$$

Substituting (9) into (6) we obtain

$$
\begin{gathered}
2 \mu^{3} h^{2} h_{z z z}+8 \mu^{3} h h_{z} h_{z z}+2 \mu^{3}\left(h_{z}\right)^{3}+3 \mu h^{2} h_{z} \\
-2 \mu h h_{z}-\lambda h_{z}=0 .
\end{gathered}
$$

Integrating this equation once with respect to $z$, we obtain

$$
2 \mu^{3} h^{2} h_{z z}+2 \mu^{3} h\left(h_{z}\right)^{2}+\mu h^{3}-\mu h^{2}-\lambda h+k_{1}=0 .
$$

The reduced ODE (11) admits the group corresponding to the generator $\mathbf{w}=\partial_{z}$.

Taking into account the invariants of its first prolongation and using the new variables

$$
\begin{aligned}
h & =\zeta, \\
h^{\prime} & =v(\zeta), \\
h^{\prime \prime} & =v(\zeta) \frac{d v}{d \zeta},
\end{aligned}
$$

we obtain that (11) can be reduced to the first-order ODE

$$
2 \mu^{3} \zeta^{2} v v_{\zeta}+2 \mu^{3} \zeta v^{2}+\mu \zeta^{3}-\mu \zeta^{2}-\lambda \zeta=0,
$$

whose implicit solution is

$$
12 \mu^{3} \zeta^{2} v^{2}+3 \mu \zeta^{4}-4 \mu \zeta^{3}-6 \lambda \zeta^{2}+12 k_{1} \zeta+k_{2}=0
$$

where $k_{1}$ and $k_{2}$ are arbitrary constants.

Applying the same procedure to (3) we get generators $\mathbf{v}_{\mathbf{1}}$ and $\mathbf{v}_{\mathbf{2}}$. The similarity variable and similarity solution are given by (9) and the corresponding reduced ODE is

$$
2 \mu^{3} h h_{z z z}+6 \mu^{3} h_{z} h_{z z}+3 \mu h^{2} h_{z}-2 \mu h h_{z}-\lambda h_{z}=0 .
$$

Integrating this equation once with respect to $z$, we obtain

$$
k+2 \mu^{3} h h_{z z}+2 \mu^{3}\left(h_{z}\right)^{2}+\mu h^{3}-\mu h^{2}-\lambda h=0 .
$$

The reduced equation (16) is an ODE that admits the symmetry generator $\mathbf{w}=\partial_{z}$. Taking into account the invariants of its first prolongation and using variables (12), we obtain the first-order ODE

$$
2 \mu^{3} \zeta v v_{\zeta}+2 \mu^{3} v^{2}+k_{1}+\mu \zeta^{3}-\mu \zeta^{2}-\lambda \zeta=0
$$


whose implicit solution is

$$
\begin{aligned}
& 60 \mu^{3} \zeta^{2} v^{2}+30 \zeta^{2} k_{1}+12 \mu \zeta^{5}-15 \mu \zeta^{4}-20 \lambda \zeta^{3}+k_{2} \\
& \quad=0
\end{aligned}
$$

where $k_{1}$ and $k_{2}$ are arbitrary constants.

2.1. Qualitative Study of ODEs. Equations (11) and (16) can be written, setting $k_{1}=0$, as

$$
\begin{aligned}
& h^{\prime \prime}+\frac{\left(h^{\prime}\right)^{2}}{h}+\frac{h}{2 \mu^{2}}-\frac{1}{2 \mu^{2}}-\frac{\lambda}{2 \mu^{3} h}=0, \\
& h^{\prime \prime}+\frac{\left(h^{\prime}\right)^{2}}{h}+\frac{h^{2}}{2 \mu^{2}}-\frac{h}{2 \mu^{2}}-\frac{\lambda}{2 \mu^{3}}=0,
\end{aligned}
$$

respectively.

Making the change of variables $x=h$ and $y=h^{\prime} h$, (19) and (20) become a system of the form

$$
\begin{aligned}
& \dot{x}=\frac{y}{x}, \\
& \dot{y}=g(x)
\end{aligned}
$$

with

$$
\begin{aligned}
& g(x)=-\frac{1}{2 \mu^{2}} x^{2}+\frac{1}{2 \mu^{2}} x+\frac{\lambda}{2 \mu^{3}}, \\
& g(x)=-\frac{1}{2 \mu^{2}} x^{3}+\frac{1}{2 \mu^{2}} x^{2}+\frac{\lambda}{2 \mu^{3}} x
\end{aligned}
$$

respectively.

The phase portrait is split into two invariant half planes $x>0$ and $x<0$. System (21) is conservative, since the quantities defined by the differentiable functions $E$

$$
\begin{aligned}
& E(x, y)=\frac{y^{2}}{2}+\frac{x^{4}}{8 \mu^{2}}-\frac{x^{3}}{6 \mu^{2}}-\frac{\lambda x^{2}}{4 \mu^{2}}, \\
& E(x, y)=\frac{y^{2}}{2}+\frac{x^{5}}{10 \mu^{2}}-\frac{x^{4}}{8 \mu^{2}}-\frac{\lambda x^{3}}{6 \mu^{3}}
\end{aligned}
$$

are constant on the trajectories; that is, $d E / d t=E_{x} \dot{x}+E_{y} \dot{y}=$ 0 . Hence the trajectories lie on the curves $E(x, y)=$ constant and they are symmetric with respect to the $x$-axis. Note that

$$
E(x, y)=\frac{y^{2}}{2}+\widetilde{U}(x),
$$

where

$$
\widetilde{U}(x)=-\int_{0}^{x} s g(s) d s
$$

The equilibrium points $P$ of (21), if any, all lie on the $x$-axis and correspond to the critical points of $E(x, y)$, since

$$
\begin{aligned}
& \frac{\partial E}{\partial x}=-x g(x)=0 \\
& \Longleftrightarrow \dot{y}=0, \\
& \frac{\partial E}{\partial y}=y=0 \\
& \Longleftrightarrow \dot{x}=0 .
\end{aligned}
$$

$P\left(x^{*}, 0\right)$ is a fixed point of $(21)$ if $x^{*}$ is a critical point of $\widetilde{U}(x)$, that is, a zero of the polynomial function $g(x)(22)$ and (23). We can set down the following.

Theorem 1. If $x^{*}$ is a strict local maximum of the analytic function $\widetilde{U}(x)$, then $P$ is a saddle for (21) if $x^{*}>0$ and a center for (21) if $x^{*}<0$. If $x^{*}$ is a strict local minimum of the analytic function $\widetilde{U}(x)$, then $P$ is a center for (21) if $x^{*}>0$ and a saddle for (21) if $x^{*}<0$. If $x^{*}$ is a horizontal inflection point of $\widetilde{U}(x)$ then $P$ is a cusp for system (21).

Proof. In fact the Jacobian matrix of the linearized system (21) at $P\left(x^{*}, 0\right)$ is

$$
J(P)=\left[\begin{array}{cc}
0 & \frac{1}{x^{*}} \\
g^{\prime}\left(x^{*}\right) & 0
\end{array}\right] .
$$

Let $\Delta=\operatorname{det}(J(P))=-g^{\prime}(x) / x$ be its determinant value. The eigenvalues of the characteristic polynomial, $p(\delta)$, of $J$ are of the form

$$
\delta= \pm \sqrt{-\Delta}
$$

On the other hand, the second derivative of $\widetilde{U}$ is

$$
\widetilde{U}^{\prime \prime}(x)=-g(x)-x g^{\prime}(x) .
$$

So $\widetilde{U}^{\prime \prime}\left(x^{*}\right)=-x^{*} g^{\prime}\left(x^{*}\right)$. Note that since the signs of $x^{*} g^{\prime}\left(x^{*}\right)$ and $g^{\prime}\left(x^{*}\right) / x^{*}$ are the same, then the difference between the sign of $\widetilde{U}^{\prime \prime}(x)$ and $\Delta$ depends on the sign of $x$. According to this, if $x^{*}$ is a local maximum of $\widetilde{U}(x)$, that is, $\widetilde{U}^{\prime \prime}\left(x^{*}\right)<0$, then $\Delta<0$ if $x^{*}>0$, and by (29), $P$ is a saddle for system (21). If $x^{*}<0$, then $\Delta>0$, and by (29), $P$ is a center for the linearized system of (21). Consider

$$
\left[\begin{array}{l}
\dot{x} \\
\dot{y}
\end{array}\right]=J\left[\begin{array}{l}
x \\
y
\end{array}\right] .
$$

So $P$ is either a center or a focus for (21). But since both, attractive fixed points and repellers, cannot occur in a conservative system [25], $P$ results in a nonlinear center for system (21).

Analogously, if $x^{*}$ is a local minimum of $\widetilde{U}(x)$, that is, $\widetilde{U}^{\prime \prime}\left(x^{*}\right)>0$, then $\Delta<0$ if $x^{*}<0$, and by (29), $P$ is a saddle for system (21). If $x^{*}>0$, then $\Delta>0$, and by (29), $P$ is a center for 
the linearized system (31) and so a nonlinear center for system (21). Finally, if $x$ is a horizontal inflection point of $\widetilde{U}(x)$, that is, $\widetilde{U}^{\prime \prime}\left(x^{*}\right)=0$, then $\Delta=0$, and by (29), $P$ is a cusp for system (21).

\section{Multipliers Method}

In [8] Anco and Bluman gave a general treatment of a direct conservation law method for partial differential equations expressed in a standard Cauchy-Kovaleskaya form in particular for evolution equations:

$$
u_{t}=G\left(x, u, u_{x}, u_{x x}, \ldots, u_{n x}\right) .
$$

The nontrivial conservation laws are characterized by a multiplier $\lambda$ with no dependence on $u_{t}$ satisfying

$$
\widehat{E}[u]\left(\Lambda u_{t}-\Lambda G\left(x, u, u_{x}, u_{x x}, \ldots, u_{n x}\right)\right)=0 .
$$

Here

$$
\widehat{E}[u]:=\frac{\partial}{\partial u}-D_{t} \frac{\partial}{\partial u_{t}}-D_{x} \frac{\partial}{\partial u_{x}}+D_{x}^{2} \frac{\partial}{\partial u_{x x}}+\cdots .
$$

The conserved current must satisfy

$$
\Lambda=\widehat{E}[u] \Phi^{t}
$$

and the flux $\Phi^{x}$ is given by [26]

$$
\Phi^{x}=-D_{x}^{-1}(\Lambda G)-\frac{\partial \Phi^{t}}{\partial u_{x}} G+G D_{x}\left(\frac{\partial \Phi^{t}}{\partial u_{x x}}\right)+\cdots
$$

The conservation law will be written as

$$
D_{t}\left(\Phi^{t}\right)+D_{x}\left(\Phi^{x}\right)=0 .
$$

We get the the following multipliers:

For (2),

$$
\begin{aligned}
& \Lambda=1, \\
& \Lambda=u, \\
& \Lambda=u^{2} u_{x x}+\frac{u^{3}}{2}-\frac{u^{2}}{2}+u u_{x}^{2} .
\end{aligned}
$$

For (3),

$$
\begin{gathered}
\Lambda=1, \\
\Lambda=u^{2} .
\end{gathered}
$$

The Subclass of Nonlinearly Self-Adjoint Equations. Equation (41) is said to be nonlinearly self-adjoint if the equation obtained from its adjoint equation by the substitution $v=$ $h\left(x, t, u, u_{x}, \ldots\right)$, with a certain function $h\left(x, t, u, u_{x}, \ldots\right)$ such that $h\left(x, t, u, u_{x}, \ldots\right) \neq 0$, is identical to the original equation (41); that is,

$$
F_{\mid v=h}^{*}=\lambda F .
$$

Equation (2) is written in the form

$$
F \equiv u_{t}+\left(u^{3}-u^{2}\right)_{x}+\left[u\left(u^{2}\right)_{x x}\right]_{x}=0 .
$$

The adjoint equation to (41) has the form

$$
F^{*} \equiv \frac{\delta(v F)}{\delta u}=0,
$$

where

$$
\frac{\delta}{\delta u}=\frac{\partial}{\partial u}-D_{t}\left(\frac{\partial}{\partial u_{t}}\right)-D_{x}\left(\frac{\partial}{\partial u_{x}}\right)+D_{x}^{2}\left(\frac{\partial}{\partial u_{x x}}\right)
$$

denotes the variational derivatives (the Euler-Lagrange operator) and $v$ is a new dependent variable. Here $D_{t}$ and $D_{x}$ are the total differentiations.

Let us single out nonlinearly self-adjoint equations from the equations of form (2). We assume that

$$
\begin{gathered}
F^{*}-\lambda\left(u_{t}+\left(u^{3}-u^{2}\right)_{x}+\left[u\left(u^{2}\right)_{x x}\right]_{x}\right) \\
-\mu\left(u_{t}+\left(u^{3}-u^{2}\right)_{x}+\left[u\left(u^{2}\right)_{x x}\right]_{x}\right)_{x} \\
-\eta\left(u_{t}+\left(u^{3}-u^{2}\right)_{x}+\left[u\left(u^{2}\right)_{x x}\right]_{x}\right)_{x x}=0,
\end{gathered}
$$

where $\lambda, \mu$, and $\eta$ are undetermined coefficients. Setting $v=h\left(x, t, u, u_{x}, u_{x x}\right)$, from the coefficients for the different derivatives of $u$, we obtain that the following conditions must be satisfied:

$$
\begin{aligned}
& \lambda=-h_{u}, \\
& \mu=-h_{u_{x}}, \\
& \eta=-h_{u_{x x}}
\end{aligned}
$$

and solving the remaining equations we get

$$
h=k_{1} u^{2} u_{x x}+k_{1} u u_{x}^{2}+c(u) u_{x}+d(u)
$$

with

$$
\begin{aligned}
& c(u)=k_{2} u^{4 / 3}, \\
& d(u)=\frac{1}{2}\left(k_{1} u^{3}-k_{1} u^{2}\right)+k_{3} u+k_{4} .
\end{aligned}
$$

We can state the following result.

(i) Equation (2) is nonlinearly self-adjoint with

$$
\begin{aligned}
& h=1, \\
& h=u, \\
& h=u^{2} u_{x x}+\frac{u^{3}}{2}-\frac{u^{2}}{2}+u u_{x}^{2} .
\end{aligned}
$$

Applying the same procedure to (3) we obtain the following result. 
(ii) Equation (3) is nonlinearly self-adjoint with

$$
\begin{aligned}
& h=1, \\
& h=u^{2} .
\end{aligned}
$$

We point out that the substitutions $h\left(x, t, u, u_{x}, u_{x x}\right)$ derived by using the condition of nonlinear self-adjointness correspond to the multipliers of Anco and Bluman method proposed in [8].

\section{Conservation Laws}

Associated to the multipliers, from (35) and (36), we obtain the corresponding conserved densities and fluxes. For (2),

(1)

$$
\begin{aligned}
\Lambda & =1, \\
\phi^{t} & =u, \\
\phi^{x} & =u\left(u^{2}+\left(-1+2 u_{x x}\right) u+2 u_{x}^{2}\right) ;
\end{aligned}
$$

(2)

$$
\begin{aligned}
\Lambda & =u, \\
\phi^{t} & =\frac{u^{2}}{2}, \\
\phi^{x} & =\frac{3}{4} u^{4}-\frac{2}{3} u^{3}+2 u^{3} u_{x x}+u^{2} u_{x}^{2} ;
\end{aligned}
$$

(3)

$$
\begin{aligned}
\Lambda & =u^{2} u_{x x}+\frac{u^{3}}{2}-\frac{u^{2}}{2}+u u_{x}^{2}, \\
\phi^{t} & =-\frac{1}{2} u_{2}^{2} u^{2}+\frac{1}{8} u^{4}-\frac{1}{6} u^{3}, \\
\phi^{x} & =\frac{1}{4}\left(u^{4}+\left(4 u_{x x}-2\right) u^{3}\right. \\
& \left.+\left(4 u_{x}^{2}+4\left(u_{x x}-\frac{1}{2}\right)^{2}\right) u^{2}\right) \\
& +\frac{1}{4}\left(8\left(u_{x x}-\frac{1}{2}\right) u_{x}^{2} u+4 u_{x}^{4}+4 u_{t} u_{x}\right) u^{2} .
\end{aligned}
$$

For (3), we get the following multipliers and the corresponding conserved densities and fluxes:

(4)

$$
\begin{aligned}
\Lambda & =1, \\
\phi^{t} & =u, \\
\phi^{x} & =2 u u_{x x}+2\left(u_{x}\right)^{2}-u^{3}+u^{2} ;
\end{aligned}
$$

(5)

$$
\begin{aligned}
\Lambda & =u^{2}, \\
\phi^{t} & =\frac{u^{3}}{3}, \\
\phi^{x} & =\frac{1}{10} u^{3}\left(20 u_{x x}+6 u^{2}-5 u\right) .
\end{aligned}
$$

By applying the theorem on conservation laws proved in [9], which avoids the integrals of functions, provided by the generators $\mathbf{v}_{\mathbf{1}}$ and $\mathbf{v}_{\mathbf{2}}$, we obtain trivial conservation laws. We point out that a method to derive conservation laws associated with scaling symmetries has been derived in [27].

\section{Conclusions}

We have applied Lie classical method to two PDEs admitting compacton solutions and nonlinear dispersion. These equations admit symmetries which lead to further reductions to first-order ODEs. They are suitable for qualitative analysis and their dynamical behaviour is described.

We have proved that (2) and (3) are nonlinearly selfadjoint. We observe that for translations generators the conservation laws via nonlinear self-adjointness method, constructed by the formulae in theorem on conservation laws [9] which avoids the integrals of functions, yield some trivial conservation laws; however by the multipliers method we have obtained some nontrivial conservation laws via integral formulae.

\section{Conflict of Interests}

The authors declare that there is no conflict of interests regarding the publication of this paper.

\section{Acknowledgments}

The authors warmly thank the referee for many valuable comments and suggestions which served to improve the paper. The support of DGICYT Project MTM2009-11875, FEDER funds, and Junta de Andalucía FQM-201 Group is gratefully acknowledged.

\section{References}

[1] P. Rosenau and J. M. Hyman, "Compactons: solitons with finite wavelength," Physical Review Letters, vol. 70, no. 5, pp. 564-567, 1993.

[2] M. S. Bruzón and M. L. Gandarias, "Traveling wave solutions of the $K(m, n)$ equation with generalized evolution," Mathematical Methods in the Applied Sciences, 2010.

[3] M. S. Bruzón, M. L. Gandarias, G. A. González, and R. Hansen, "The $K(m, n)$ equation with generalized evolution term studied by symmetry reductions and qualitative analysis," Applied Mathematics and Computation, vol. 218, no. 20, pp. 10094-10105, 2012. 
[4] R. Naz, "Conservation laws for some compacton equations using the multiplier approach," Applied Mathematics Letters, vol. 25, no. 3, pp. 257-261, 2012.

[5] P. Rosenau and A. Oron, "On compactons induced by a nonconvex convection," Communications in Nonlinear Science and Numerical Simulation, vol. 19, no. 5, pp. 1329-1337, 2014.

[6] M. L. Gandarias, "Conservation laws for some equations that admit compacton solutions induced by a non-convex convection," Journal of Mathematical Analysis and Applications, vol. 430, no. 2, pp. 695-702, 2015.

[7] S. C. Anco and G. Bluman, "Direct construction of conservation laws from field equations," Physical Review Letters, vol. 78, no. 15, pp. 2869-2873, 1997.

[8] S. C. Anco and G. Bluman, "Direct construction method for conservation laws of partial differential equations. Part II: general treatment," European Journal of Applied Mathematics, vol. 41, pp. 567-585, 2002.

[9] N. H. Ibragimov, "A new conservation theorem," Journal of Mathematical Analysis and Applications, vol. 333, no. 1, pp. 311328, 2007 .

[10] N. H. Ibragimov, "Quasi-self-adjoint differential equations," Archives of ALGA, vol. 4, pp. 55-60, 2007.

[11] M. L. Gandarias, "Weak self-adjoint differential equations," Journal of Physics A: Mathematical and Theoretical, vol. 44, no. 26, Article ID 262001, 2011.

[12] N. H. Ibragimov, "Nonlinear self-adjointness and conservation laws," Journal of Physics A: Mathematical and Theoretical, vol. 44, no. 43, Article ID 432002, 2011.

[13] M. S. Bruzón, M. L. Gandarias, and N. H. Ibragimov, "Selfadjoint sub-classes of generalized thin film equations," Journal of Mathematical Analysis and Applications, vol. 357, no. 1, pp. 307-313, 2009.

[14] I. L. Freire and J. C. S. Sampaio, "Nonlinear self-adjointness of a generalized fifth-order KdV equation," Journal of Physics A: Mathematical and Theoretical, vol. 45, no. 3, Article ID 032001, 2012.

[15] I. L. Freire, "Self-adjoint sub-classes of third and fourth-order evolution equations," Applied Mathematics and Computation, vol. 217, no. 22, pp. 9467-9473, 2011.

[16] I. L. Freire and J. C. Santos Sampaio, "On the nonlinear self-adjointness and local conservation laws for a class of evolution equations unifying many models," Communications in Nonlinear Science and Numerical Simulation, vol. 19, no. 2, pp. 350-360, 2014.

[17] N. H. Ibragimov, M. Torrisi, and R. Tracina, "Quasi self-adjoint nonlinear wave equations," Journal of Physics: A: Mathematical and Theoretical, vol. 43, no. 44, Article ID 442001, 2010.

[18] N. H. Ibragimov, M. Torrisi, and R. Tracinà, "Self-adjointness and conservation laws of a generalized Burgers equation," Journal of Physics A: Mathematical and Theoretical, vol. 44, no. 14, Article ID 145201, 2011.

[19] M. L. Gandarias, "Weak self-adjointness and conservation laws for a porous medium equation," Communications in Nonlinear Science and Numerical Simulation, vol. 17, no. 6, pp. 2342-2349, 2012.

[20] M. L. Gandarias, M. Redondo, and M. S. Bruzón, "Some weak self-adjoint Hamilton-Jacobi-Bellman equations arising in financial mathematics," Nonlinear Analysis: Real World Applications, vol. 13, no. 1, pp. 340-347, 2012.

[21] M. L. Gandarias and M. S. Bruzó, "Some conservation laws for a forced KdV equation," Nonlinear Analysis:Real World Applications, vol. 13, no. 6, pp. 2692-2700, 2012.
[22] L. R. Galiakberova and N. H. Ibragimov, "Nonlinear selfadjointness of the Krichever-Novikov equation," Communications in Nonlinear Science and Numerical Simulation, vol. 19, no. 2, pp. 361-363, 2014

[23] M. L. Gandarias, "Nonlinear self-adjointness through differential substitutions," Communications in Nonlinear Science and Numerical Simulation, vol. 19, no. 10, pp. 3523-3528, 2014.

[24] M. Torrisi and R. Tracinà, "Quasi self-adjointness of a class of third order nonlinear dispersive equations," Nonlinear Analysis: Real World Applications, vol. 14, no. 3, pp. 1496-1502, 2013.

[25] S. H. Strogatz, Nonlinear Dynamics and Chaos, Perseus Publishing, Cambridge, Mass, USA, 1994.

[26] N. Euler and M. Euler, "On nonlocal symmetries, nonlocal conservation laws and nonlocal transformations of evolution equations: two linearisable hierarchies," Journal of Nonlinear Mathematical Physics, vol. 16, no. 4, pp. 489-504, 2009.

[27] S. C. Anco, "Conservation laws of scaling-invariant field equations," Journal of Physics A: Mathematical and General, vol. 36, no. 32, pp. 8623-8638, 2003. 


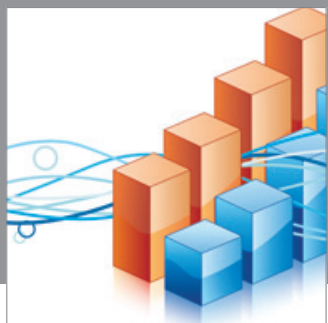

Advances in

Operations Research

mansans

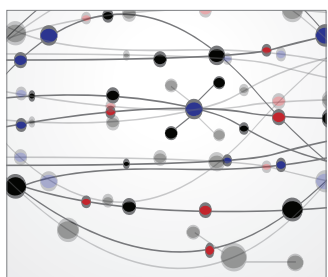

The Scientific World Journal
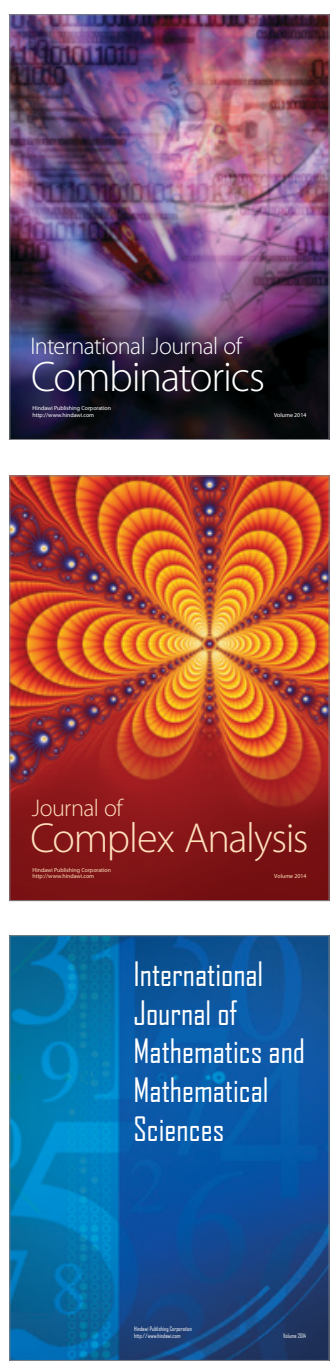
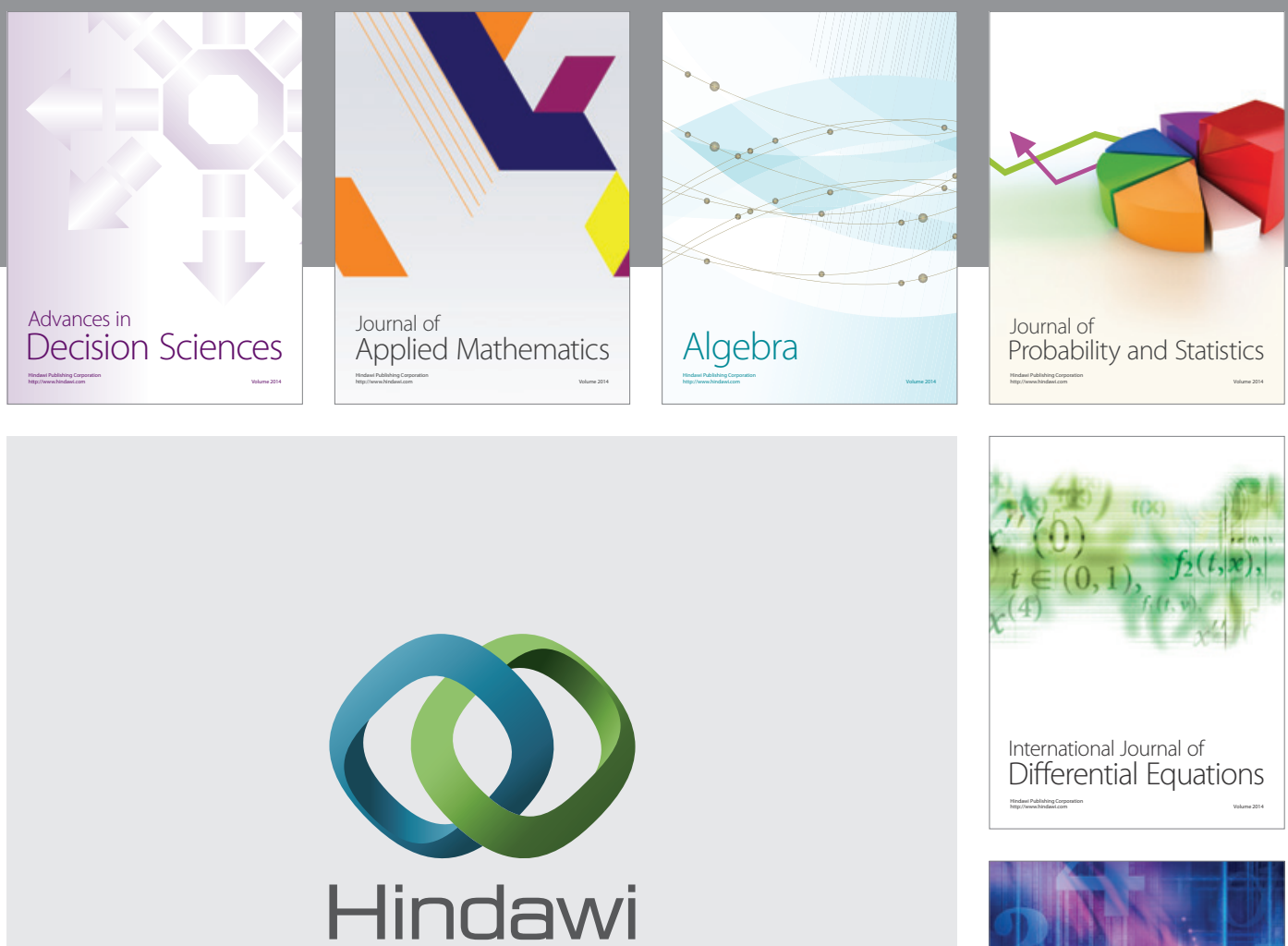

Submit your manuscripts at http://www.hindawi.com
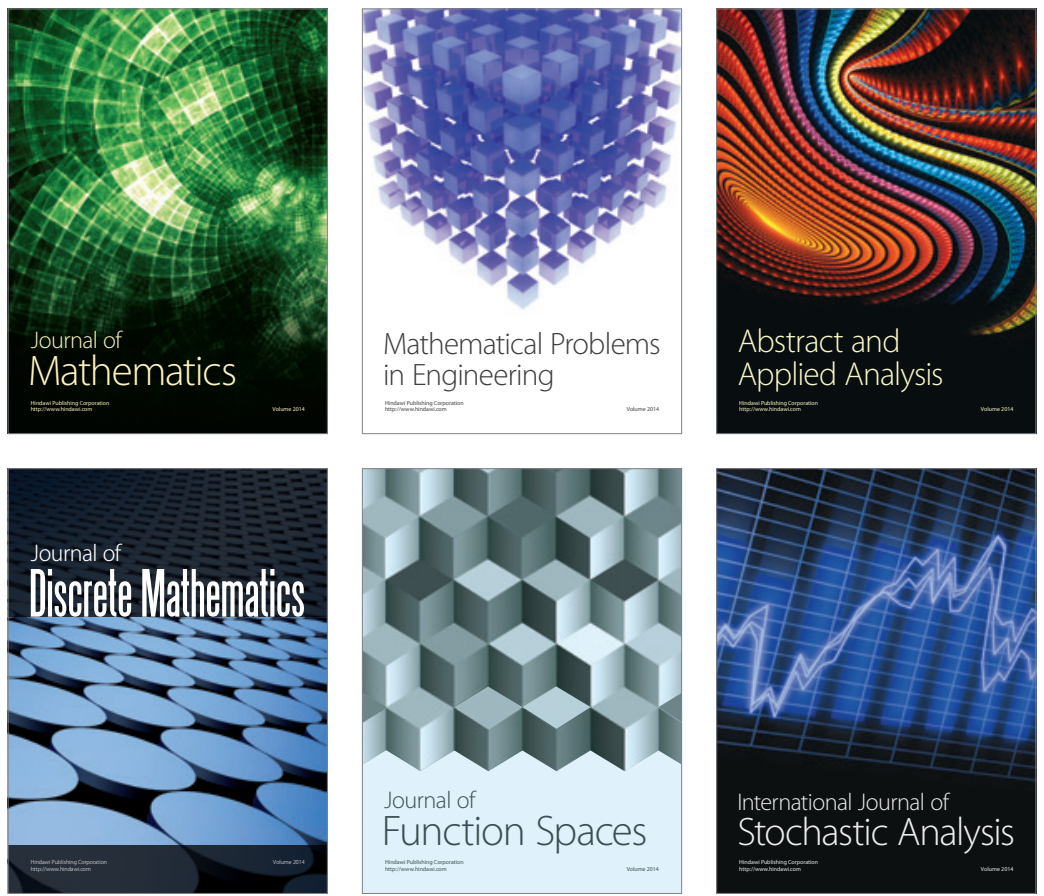

Journal of

Function Spaces

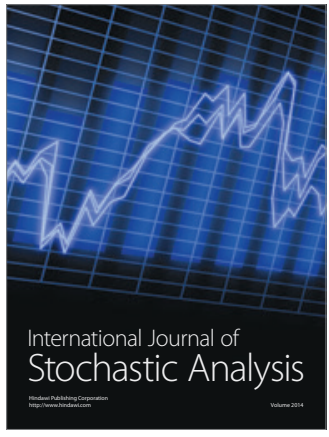

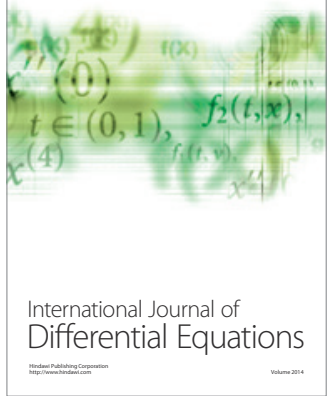
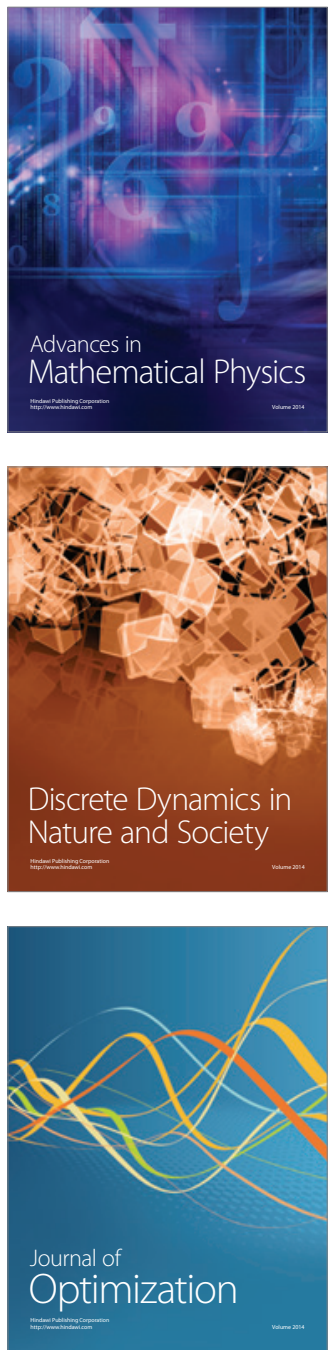